\title{
Transformative science unlocked by future geodetic data at Mars, Venus, and Ocean Worlds
}

\author{
A white paper submitted to the 2023-2032 Decadal Survey on \\ Planetary Science and Astrobiology
}

\section{Primary Author:}

Michael M. Sori, Purdue University

954-632-9860, msori@purdue.edu

\section{Co-authors:}

Anton I. Ermakov, University of California-Berkeley

James Tuttle Keane, NASA Jet Propulsion Laboratory

Bruce G. Bills, NASA Jet Propulsion Laboratory

Ali M. Bramson, Purdue University

Alexander J. Evans, Brown University

Douglas J. Hemingway, Carnegie Institution for Science

Peter B. James, Baylor University

Brandon C. Johnson, Purdue University

Harriett C.P. Lau, University of California-Berkeley

Thomas Navarro, UCLA/McGill University

Joseph G. O'Rourke, Arizona State University

Lujendra Ojha, Rutgers University

Ryan S. Park, NASA Jet Propulsion Laboratory

David E. Smith, Massachusetts Institute of Technology

Krista M. Soderlund, University of Texas

David A. Spencer, Purdue University/NASA Jet Propulsion Labatory

Sonia M. Tikoo, Stanford University

Renee C. Weber, NASA Marshall Space Flight Center

Maria T. Zuber, Massachusetts Institute of Technology

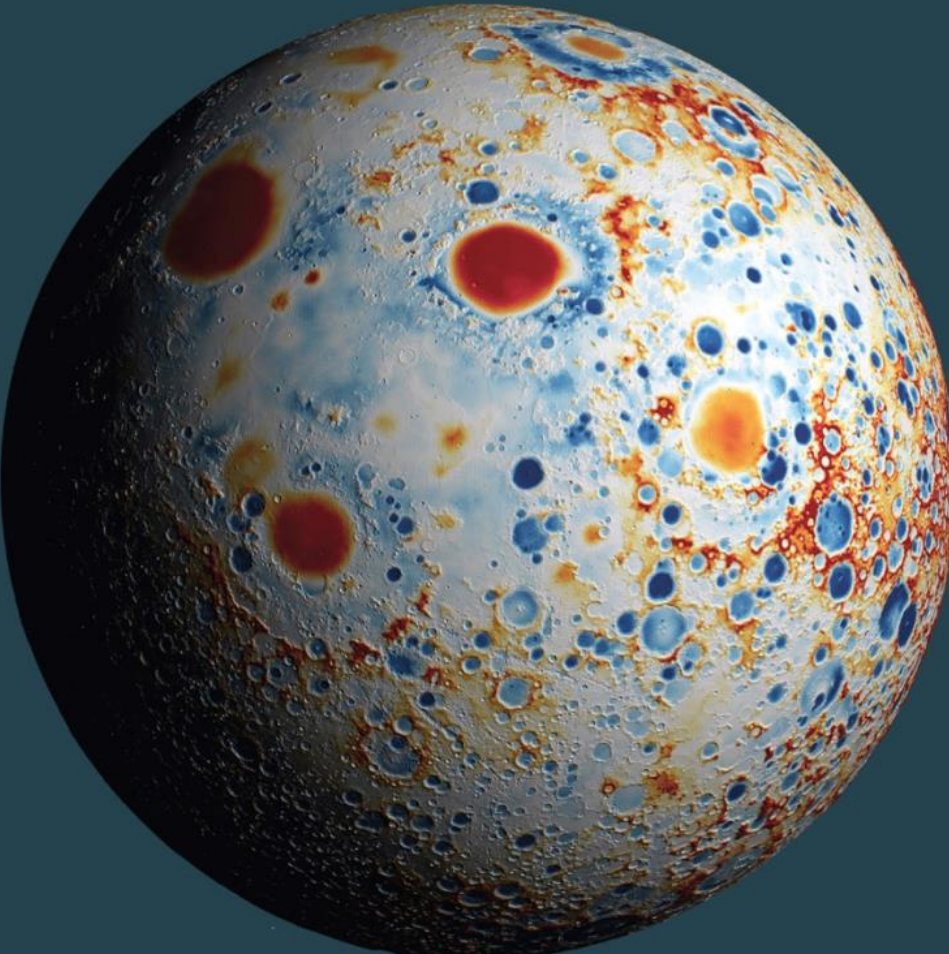

Cover image shows the static gravity fields of (in decreasing order of size) the Moon, Earth, Mars, Venus, and Enceladus. The size of each body is scaled by the quality of the gravity data available at that world. 


\section{Why: Geodetic missions can address priority questions in planetary science}

While geodesy in the Earth-Moon system has flourished in the past decade, geodesy at other worlds has lagged behind (see Fig. 1, which shows current geodetic data resolution in spherical harmonics1). Closing this gap by elevating geodetic measurements across the Solar System to the level of those taken in the Earth-Moon system would enable fundamental leaps in planetary science in a multitude of disciplines. In this paper, we describe diverse and transformative science that would be enabled by new gravity and topography data. On this basis, we advocate for the focused collection of geodetic data across the solid bodies of the Solar System with new spacecraft missions.

Geodesy - the study of a world's shape, orientation, and gravity field-is one of the most powerful methods for investigating the formation, evolution, structure, and active processes of Solar System bodies. The power of geodesy is best demonstrated in the Earth-Moon system, where high-precision measurements have transformed geodesy from a purely geophysical tool into one that unlocks advances in geology, hydrology, climate, atmospheric science, geochemistry, and more. For example, measurements of Earth's time-variable gravity field from the NASA GRACE, GRACE Follow-On, and the ESA GOCE missions have enabled study of dynamic processes like hydrological cycles and climate change. At the Moon, geodetic measurements of topography from the LOLA instrument aboard NASA's LRO mission have provided an essential reference frame for other orbital datasets in addition to directly enabling science, and measurements of gravity to high precision from NASA's GRAIL mission have provided unprecedented insights into the formation and evolution of planetary crusts. While some topography and gravity data across the Solar System have revealed insights into other planets and moons, substantial gaps in our knowledge remain. Dedicated geodetic missions in the next decade would dramatically advance high-priority questions in planetary science.

\section{Where: The most promising targets for the next decade}

We focus on the following targets: Mars, Venus, and icy satellites that host subsurface oceans (e.g., Europa, Enceladus, Titan; hereafter called "Ocean Worlds" or OWs). These bodies are feasible targets for focused geodetic missions, have broad appeal within the planetary science community, and are destinations where new geodetic measurements would enable transformative planetary science. At Mars and Venus, we focus on the collection of new gravity data from orbit, as knowledge of the gravity field currently severely limits geophysical analyses (compared to knowledge of global topography). At Ocean Worlds, we consider collection of both topography and gravity data to be high priority. We focus on data collection at each individual body, although we note that geodetic measurements between planets has value and is described in a separate report [Smith et al., 2018]. In this report, we demonstrate that geodetic data is critical to addressing scientific objectives outlined by the Mars Exploration Program Analysis Group

${ }^{1}$ Spherical harmonic coefficients describe a function on a sphere, and are used here to describe the resolution to which a planetary gravity or topography field is known. Higher spherical harmonic degrees correspond to smaller spatial scales and higher resolution, and vice-versa. See Wieczorek [2015] for a review. 
(MEPAG), the Venus Exploration Analysis Group (VEXAG), the Outer Planets Assessment Group (OPAG), the Mars Next Orbiter Science Analysis Group (NEXSAG), and the Mars Ice and Climate Evolution Science Analysis Group (ICESAG).

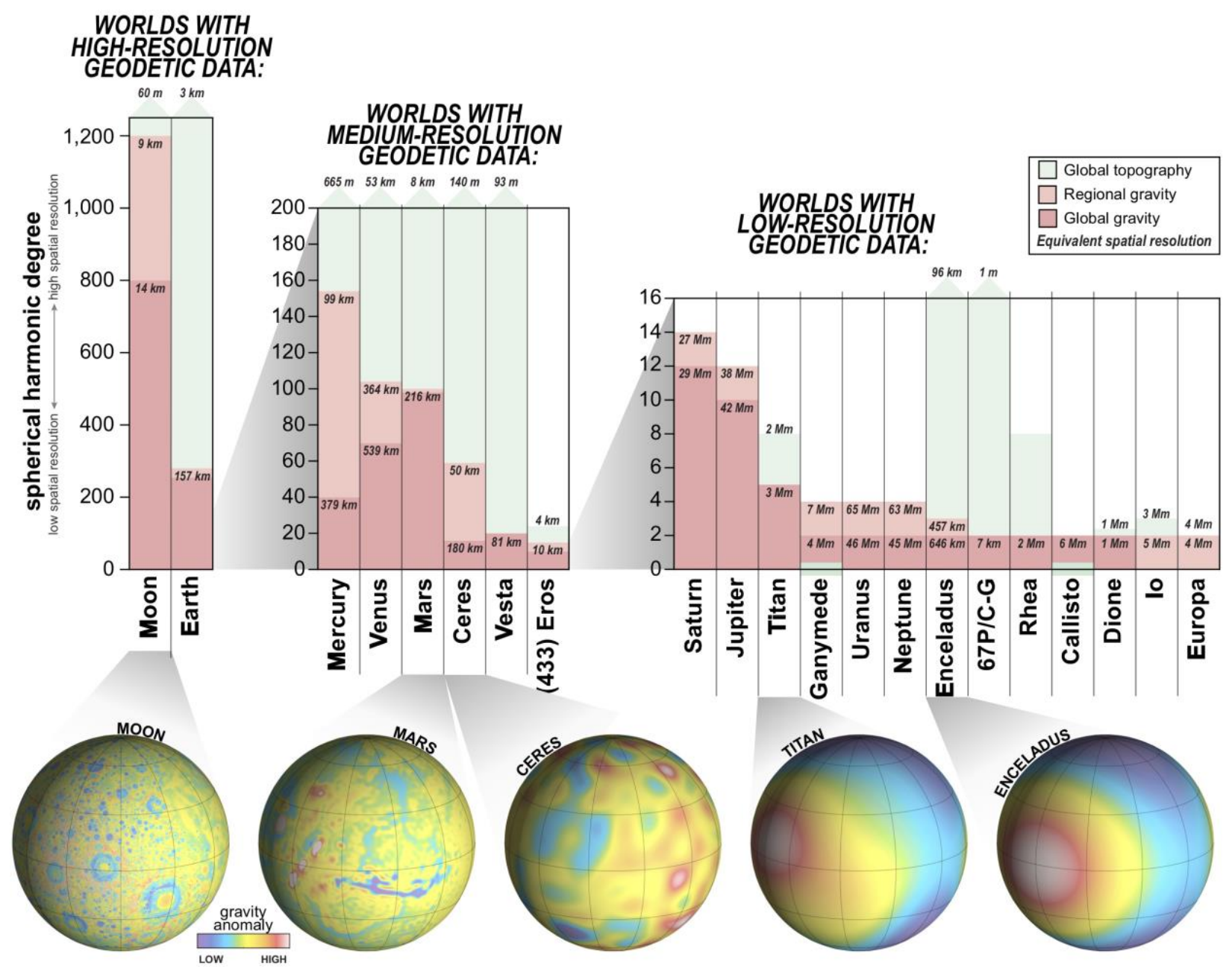

Figure 1. Current knowledge of topography and gravity across the Solar System from orbital spacecraft, for planets, moons, and small bodies for which gravity data beyond total mass is known. Resolution plotted in terms of spherical harmonic degree and labeled with text of the equivalent spatial resolution (in meters, kilometers, or megameters).

\section{What: New science unlocked at Mars, Venus, and Ocean Worlds}

\subsection{Mars}

Gravity science at Mars can be used to answer: Why and how do terrestrial planets evolve differently? Current knowledge of the Martian gravity field is insufficient to satisfyingly address this question. The gravity field at Mars has been inferred from Doppler tracking of multiple orbiting spacecraft using NASA's Deep Space Network (DSN). These observations have allowed for the construction of a gravity field with degree strength of $\sim 100$, corresponding to a wavelength of $213 \mathrm{~km}$, although it is not globally 
uniform [Genova et al., 2016]. The gravity field is updated incrementally as more tracking data of Mars-orbiting spacecraft are acquired, but the next fundamental leap in using gravity science at Mars to understand terrestrial planet evolution would require a dedicated mission similar to GRAIL, GRACE, or GOCE.

A dedicated gravity mapping mission would allow for the geophysical analysis of the Martian shallow and deep interior in unprecedented detail. A dedicated Mars geodesy mission would enable major scientific advances including quantifying the inner Solar System's early impact bombardment through searches of buried craters, quantifying the relative contribution of igneous and sedimentary processes, elucidating the origin of the crustal dichotomy and crustal magnetization, exploring the plumbing of Mars's gigantic volcanoes, and more. At greater depths, gravity data could be used to constrain core size. At both shallow and deep depths, gravity analysis would be complementary to InSight's geophysical investigations, increasing the scientific return of both datasets. Thus, new gravity science measurements at Mars are especially timely for the next decade.

High-precision gravity data at Mars would enable exciting advances in comparative climatology of terrestrial worlds. Mars represents a natural laboratory to study how climate evolves in response to astronomical variations, which are more extreme on Mars than on Earth. At the poles, high-precision knowledge of the static gravity field would allow for robust constraints on the volume of carbon dioxide and dust in the polar layered deposits, determination of the loading history of the polar regions, and the mapping of circumpolar ice deposits. The time-variable gravity field, if acquired over several years, would allow for real-time observations of the active accumulation or sublimation of the polar layered deposits, thereby determining their present-day annual deposition rates-one of the most important questions in Mars polar science.

Perhaps most unique to Mars over the next decade is that gravity data would serve a key role in paving the way for human exploration. An important resource for human explorers will be accessible water. Therefore, it is essential to map near-surface water ice in the regions where human landing would most likely occur, but debate persists as to the extent of ice in the most promising areas [Bramson et al. 2015; Campbell and Morgan, 2018]. Gravity would be an ideal tool to use in tackling this problem, but current measurement precision is insufficient. Furthermore, characterization of the gravity field to high accuracy would support the entry, descent, and landing operations for rovers and human explorers, akin to the mapping of ocean currents for sailors.

\subsection{Venus}

Like Mars, gravity science at Venus may be used to answer: Why and how do terrestrial planets evolve differently? Venus is of similar size to Earth, but has evolved into a very dissimilar and hostile planet. Collection of gravity data from orbit would elucidate the different evolutionary paths of the two planets. At present, the Venus gravity field has degree strength 70, corresponding to a wavelength of $539 \mathrm{~km}$, although the quality of the data varies across the planet [Konopliv et al., 1999].

A critical investigation that would be enabled by orbital gravity science on Venus is determination of the nature and rates of crustal recycling. Venus' surface is geologically young, but the similarities to and differences from plate tectonics on Earth are debated. A gravity-derived inventory of impact structures on Venus would constrain the timing of resurfacing and address the long-standing debate over whether that resurfacing was 
gradual or catastrophic. Precise gravity data could be used to search for magma chambers, and density inversions would allow for compositional constraints on volcanic features, both of which would quantify the role of igneous activity. New gravity data would enable substantially improved investigations of topographic compensation, which could be used to understand crustal structure and mantle dynamics.

Venus offers an opportunity for the innovative application of gravity data to study atmospheric science of a terrestrial planet. Dynamics in the Venusian atmosphere represent a substantial movement of mass, and would be observable in realistically obtainable gravity data [Bills et al., 2020]. Diurnal variations of surface temperature and pressure driven by thermal tides would be detectable and constrain deep atmospheric dynamics, which may be otherwise inaccessible by remote sensing. Time-variable gravity could also constrain the torque of the Sun acting on the atmosphere, testing hypotheses about the origin of Venus' slow retrograde rotation.

Understanding of Venus' deep interior is limited by the low sensitivity of the gravity field to the internal structure due to Venus' slow rotation. This knowledge gap can be eliminated by measuring Venus' moment of inertia through tracking Venus' precession. A better understanding of deep interior, mantle dynamics, and dissipation would also help constrain the evolution of Venus' core, specifically, when a geodynamo would have existed. A better understanding of magnetic field evolution could then be linked to the diverging evolution of terrestrial planet atmospheres.

\subsection{Ocean Worlds}

Topography and gravity data at Ocean Worlds may be used to answer: What are their interior structures, and what are the implications of those structures for habitability? The exploration of OWs is currently in its infancy, and there are major knowledge gaps about the formation, evolution, and interior structure of these bodies. The OW for which we have the best gravity field is Titan, which comes from 10 fly-bys and is inferred only to degree 5 , corresponding to a wavelength of nearly $3,000 \mathrm{~km}$ [Durante et al., 2019]. These low-resolution data inhibit basic geophysical investigations of the interior structure of OWs, yielding substantial uncertainties in critical quantities like the thickness of the ocean. The current state of knowledge of the interior structure of OWs is comparable to the pre-Apollo view of the Moon. A similar lack of knowledge exists for long-wavelength, global topography data. At Europa, the dearth of geodetic data may be partially addressed by NASA's Europa Clipper mission, but this spacecraft will not address all objectives that could be accomplished by a dedicated geophysical mission. This knowledge gap was recognized in the recent Planetary Science Midterm Review: "[Europa Clipper] achieves many but not all of the science goals identified for the Jupiter Europa Orbiter" (page 13) and "the major loss is to the geophysical science objectives; without being in orbit it is challenging to establish the interior structure of Europa" (page 69). Thus, we argue for a broad geodetic approach at OWs over the next decade that emphasizes both topography and gravity data.

Geodetic missions would provide strong tests of the hypothesis that a particular icy moon hosts a global or regional subsurface ocean of liquid water. The presence of oceans leads to testable predictions for their host body's topography and gravity fields. Beyond providing exceptionally strong evidence for the existence of an ocean, geodetic data would enable physical characterization of that ocean. Time-variable gravity data could be 
used to study tidal effects in an ice shell and ocean, and, if sufficiently precise, to study ocean currents. It has been demonstrated that precise topography and gravity data could also be used to test the hypothesis that volcanism occurs on the ocean seafloor of bodies where there is a water-silicate interface, which might be a crucial test on whether the ocean has the energy budget to be habitable [Dombard and Sessa, 2019].

Study of time-variable shape data (i.e., surface deformation) should also be prioritized for OWs. Measurement of active deformation through methods like laser ranging and precise tracking of landed instruments would yield insight into interior structure and how tidal energy operates in the satellite of a giant planet. Precise measurement of surface deformation would also provide a strong test of the hypothesis that OWs (particularly Europa) undergo a plate tectonics-like process, where slabs of icy crust descend into an ocean [Kattenhorn and Prockter, 2014]. Such a process would represent a mechanism of surface-ocean exchange, which may be a critical ingredient for habitability.

Given the current lack of knowledge of OW interiors, a dedicated geodetic mission to any OW would yield transformative science for all OWs. A detailed study is necessary to determine which target $\mathrm{OW}$ would yield the highest science return for a given cost.

\subsection{All Worlds}

In the above sections, we focused on science that would be enabled by geodetic data at particular planetary bodies, but some essential geophysical analyses are more general and would apply to all cases. Precise topography and gravity data allow for mapping of the thickness of the silicate crust (Mars and Venus) and ice shell (OWs), especially when combined with other geological constraints. Sufficiently precise geodetic data would permit isolation of the gravitational signature of the crust or ice shell, a fundamental improvement over what is possible with current data, leading to the construction of crustal density maps. In all cases, geodetic data would allow for constraints on deep interior structure and on lithospheric thickness. All three of these scientific investigations-crustal thickness mapping, crustal density mapping, and interior structure constraints-elucidate fundamental aspects of a planet, including its formation and thermal history.

In Table 1, we list topics that geodetic data collection could address at all worlds, and how such topics map to MEPAG, NEXSAG, and ICESAG objectives (Mars), VEXAG objectives (Venus), and objectives outlined in OPAG'S Roadmap to Ocean Worlds. This list is not exhaustive for any of these planets and moons.

\section{How: Conclusions and Recommendations}

We conclude that new geodetic data at Mars, Venus, and Ocean Worlds have tremendous promise for advancing planetary science in the next decade and beyond, and would enable fundamental steps forward in our understanding of the Solar System's geophysics, habitability, geology, geochemistry, glaciology, atmospheric science, and more. We therefore advocate for the collection of such data through new spacecraft missions. In this report, we do not explicitly advocate for a specific mission architecture. However, we note that several possible concepts are realistic, and should be studied for feasibility. For the collection of topography or surface deformation data, we suggest laser ranging (e.g., involving orbital altimeters, surface retroreflectors, or both) be considered. For the collection of gravity data, spacecraft-to-spacecraft tracking (as was done at the 
Moon with GRAIL [Zuber et al., 2013] and at Earth with GRACE [Tapley et al., 2004]) and gravity gradiometry (as was done at Earth with GOCE [Drinkwater et al., 2003]) have substantial promise. The authors of this white paper are hosting a Keck Institute for Space Sciences study program to study which of these geodetic options are most promising for enabling science over the next decade.

Recommendation \#1: We recommend that the decadal survey consider geodesy as a fundamental component in the scientific investigation of the Solar System, and prioritize closing the gap between geodesy in the Earth-Moon system vs. geodesy at other planets.

Recommendation \#2: We recommend that orbital gravity science be prioritized at Mars and Venus. Next-generation gravity science at Mars and Venus may be possible with dedicated missions or as part of a larger, more all-purpose mission.

Recommendation \#3: We recommend that a geophysical orbiter that collects both global topography and gravity data be prioritized at an Ocean World. To support this plan, we recommend that the decadal survey support a mission concept study for a New Frontiers class "Ocean World Geophysical Explorer," which would include a trade study (balancing science, engineering, and cost) to determine its optimal destination (e.g., Titan, Europa, Enceladus, etc.).

Recommendation \#4: We recommend that technology development to enable planetary geodesy be prioritized, including the further development of gravity gradiometers, ranging lasers, and CubeSats and SmallSats that can conduct geodetic measurements.

Table 1. Examples of scientific investigations that would be enabled by geodetic missions at Mars (orange, this page), Venus (yellow, top next page), and Ocean Worlds (blue, middle next page). "Objectives addressed" refers to objectives of planetary exploration as listed by NASA assessment groups MEPAG (M), ICESAG (i), NEXSAG (N), VEXAG (V), and OPAG (O). Numbers and letters correspond to goals and objectives; e.g., "M2B1" refers to MEPAG Goal 2, Objective B, Sub-objective 1. Examples are not exhaustive.

\begin{tabular}{|l|l|l|}
\hline $\begin{array}{l}\text { Topic (Mars) } \\
\text { Impact history }\end{array}$ & $\begin{array}{l}\text { Objectives Addressed } \\
\text { M3B1, M3B2 }\end{array}$ & $\begin{array}{l}\text { Description } \\
\text { Inventory large basins to reconstruct } \\
\text { bombardment history }\end{array}$ \\
\hline $\begin{array}{l}\text { Water resource } \\
\text { mapping }\end{array}$ & $\begin{array}{l}\text { M2B3, M2C2, M3A1, } \\
\text { M3A4, M4D1, i8, i9, i10, } \\
\text { i13, i16, NSA, NRA }\end{array}$ & $\begin{array}{l}\text { Test for the presence of near-surface ice sheets at } \\
\text { mid-latitudes and search for other hidden water- } \\
\text { ice }\end{array}$ \\
\hline Carbon dioxide & $\begin{array}{l}\text { M2B1, M2B2, M2C1, } \\
\text { Quantify mass of carbon dioxide in polar caps } \\
\text { and thereby the planet's atmospheric reservoirs }\end{array}$ \\
\hline Active climate & $\begin{array}{l}\text { M2A1, M2A4, M2B1, } \\
\text { M2B2, i1, i4, i5, i6 }\end{array}$ & $\begin{array}{l}\text { Estimate ice accumulation rates of the polar } \\
\text { layered deposits in real time }\end{array}$ \\
\hline $\begin{array}{l}\text { Sedimentary } \\
\text { processes } \\
\text { Magnetic field } \\
\text { origin }\end{array}$ & M2B3, M2A3, i3, i6, NSD & $\begin{array}{l}\text { Constrain density and origin of possibly } \\
\text { sedimentary features, e.g. Medusa Fossae } \\
\text { Constrain deep interior to facilitate models of } \\
\text { core dynamo; constrain shallow interior to test } \\
\text { hypotheses on crustal magnetization }\end{array}$ \\
\hline
\end{tabular}




\begin{tabular}{|l|l|l|}
\hline $\begin{array}{l}\text { Topic (Venus) } \\
\text { Resurfacing history }\end{array}$ & V1RE, V3GH, V3GA & $\begin{array}{l}\text { Description } \\
\text { Inventory large basins to constrain resurfacing } \\
\text { timing and nature (catastrophic vs. gradual) }\end{array}$ \\
\hline $\begin{array}{l}\text { Atmosphere } \\
\text { characterization }\end{array}$ & V2DD, V2UD, V2MP & $\begin{array}{l}\text { Observe moving atmospheric mass to understand } \\
\text { deep atmospheric dynamics }\end{array}$ \\
\hline Mantle dynamics & V1RE, V1LI, V1HF & $\begin{array}{l}\text { Determine how topography is supported to } \\
\text { constrain mantle dynamics }\end{array}$ \\
\hline Volcanism & V3GH, V3GA, V3CR & $\begin{array}{l}\text { Test for the existence of magma chambers; } \\
\text { constrain density and composition of volcanic } \\
\text { features }\end{array}$ \\
\hline $\begin{array}{l}\text { Magnetic field } \\
\text { origin }\end{array}$ & V1MA, V1CO & $\begin{array}{l}\text { Constrain deep interior to facilitate models of } \\
\text { core dynamo }\end{array}$ \\
\hline $\begin{array}{l}\text { Rotation rate } \\
\text { Constrain net torque on Venus to test hypothesis } \\
\text { for its slow, retrograde rotation }\end{array}$ \\
\hline
\end{tabular}

\begin{tabular}{|l|l|l|}
\hline $\begin{array}{l}\text { Topic (OW) } \\
\text { Existence of oceans }\end{array}$ & $\begin{array}{l}\text { Objectives Addressed } \\
\text { Ocean currents }\end{array}$ & $\begin{array}{l}\text { Oescription } \\
\text { Onfer interior structure using gravity, topography, } \\
\text { and surface deformation measurements }\end{array}$ \\
\hline Cryovolcanism & O1B1, O1B3, O2A1, O4A2 & $\begin{array}{l}\text { Detect water movement in oceans using time- } \\
\text { variable gravity measurements }\end{array}$ \\
$\begin{array}{l}\text { Constrain possible cryovolcanic features and } \\
\text { subsurface plumbing using gravity and } \\
\text { topography measurements }\end{array}$ \\
\hline Tidal heating & O1A3, O1A4, O1B5, O1C4, & $\begin{array}{l}\text { Constrain moon response to tides using time- } \\
\text { variable topography and gravity measurements }\end{array}$ \\
\hline Icy plate tectonics & O1B1, O1B2 & $\begin{array}{l}\text { Constrain lateral movement of surface using } \\
\text { surface deformation measurements }\end{array}$ \\
\hline Seafloor volcanism & O2B2, O4A2 & $\begin{array}{l}\text { Characterize interface between water and } \\
\text { silicates using gravity and topography } \\
\text { measurements }\end{array}$ \\
\hline
\end{tabular}

\section{References}

[1] Bills, B.G., et al. (2020), lcarus 340, 113568.

[2] Bramson, A.M., et al. (2015), Geophys. Res. Lett. 42, 6566-6574.

[3] Campbell, B.A. and G.A. Morgan (2018), Geophys. Res. Lett. 45, 1759-1766.

[4] Dombard, A.J. and A.M. Sessa (2019), Icarus 325, 31-38.

[5] Drinkwater, M.R., et al. (2003), Space Sci. Rev. 108, 419-432.

[6] Durante, D., et al. (2019), lcarus 326, 123-132.

[7] Genova, A., et al. (2016), Icarus 272, 228-245.

[8] Kattenhorn, S.A. and L.M. Prockter (2014), Nature Geosci. 7, 762-767.

[8] Konopliv, A.S., et al. (1999), Icarus 139, 3-18.

[9] Smith, D.E., et al. (2018), Planet. Space Sci. 153, 127-133.

[10] Tapley, B.D., et al. (2004), Geophys. Res. Lett. 31.

[11] Wieczorek, M.A. (2015), Treatise on Geophysics 10, 153-193.

[12] Zuber, M.T., et al. (2013), Science 339, 668-671. 